\title{
AVALIAÇÃO DA IMPLANTAÇÃO DA NORMA REGULAMENTADORA 32 EM UM HOSPITAL UNIVERSITÁRIO*
}

\author{
Maikon Rosa dos Santos ${ }^{1}$, Renata Perfeito Ribeiro ${ }^{2}$, Mariana Bessa Martins ${ }^{3}$, Leonel Alves do Nascimento ${ }^{4}$, \\ Júlia Trevisan Martins ${ }^{5}$, Maria Cristina Cescatto BoBroff ${ }^{6}$
}

RESUMO: Este estudo teve como objetivo verificar a implantação da Norma Regulamentadora 32 em um hospital do Norte do Paraná. Realizou-se um estudo de caso do tipo exploratório e descritivo, por meio entrevista gravada com uma enfermeira da Comissão de Controle de Infecção Hospitalar, em junho de 2010. Percebeu-se que a instituição tem dificuldades em relação à implantação desta norma, entre elas a indisponibilidade e não utilização de equipamentos de proteção individual, estrutura física inadequada e sobrecarga nas caixas de descarte de resíduos perfurocortantes. Concluiuse que, apesar destas dificuldades, foram adotados alguns quesitos em relação à norma e instituiu-se uma comissão com o objetivo de implantá-la de acordo com a regulamentação governamental.

PALAVRAS-CHAVE: Saúde do trabalhador; Riscos ocupacionais; Exposição a agentes biológicos; Regulamentação governamental.

\section{EVALUATION OF THE IMPLANTATION OF REGULATORY NORM 32 IN A UNIVERSITY HOSPITAL}

\begin{abstract}
This study aimed to verify the implantation of Regulatory Norm 32 in a hospital in the north of the Brazilian state of Paraná. An exploratory, descriptive case study was carried out through a recorded interview with a nurse from the Hospital Infection Control Committee, in June 2010. It was noticed that the institution has difficulties in relation to the implantation of the Norm, including unavailability of, and failure to use, personal protective equipment, and inadequate physical structure and overloading of containers for the disposal of sharps. It is concluded that despite these difficulties, some aspects were adopted in relation to the norm and a commission was set up with the objective of implanting it in line with governmental regulations. KEYWORDS: Workers' health; Occupational hazards; Exposure to biological agents; Governmental regulations.

\section{EVALUACIÓN DE LA IMPLANTACIÓN DE LA NORMA REGLAMENTADORA 32 EN UN HOSPITAL UNIVERSITARIO}

RESUMEN: Este estudio tuvo como objetivo verificar la implantación de la Norma Reglamentadora 32 en un hospital de Norte de Paraná. Fue realizado un estudio de caso del tipo exploratorio y descriptivo, por medio de entrevista grabada con una enfermera de la Comisión de Control de Infección Hospitalar, en junio de 2010. Se percibió que la institución tiene dificultades para la implantación de esta norma, entre las cuales la indisponibilidad y no utilización de equipos de protección individual, estructura física inadecuada y sobrecarga en las cajas de descarte de residuos cortantes. Se concluyó que, a pesar de estas dificultades, fueron adoptados algunos criterios acerca de la norma y se instituyó una comisión con el objetivo de implantarla de acuerdo con la reglamentación gubernamental.

PALABRAS CLAVES: Salud del trabajador; Riesgos ocupacionales; Exposición a agentes biológicos; Reglamentación gubernamental.

\footnotetext{
*Artigo extraído do trabalho de conclusão do Curso de Graduação em Enfermagem apresentado em 2010 à Universidade Estadual de Londrina - UEL.

\section{${ }^{1}$ Enfermeiro.}

${ }^{2}$ Enfermeira. Mestre em Enfermagem. Doutoranda pelo Programa Interunidades da Escola de Enfermagem de Ribeirão Preto da Universidade de São Paulo.

${ }^{3}$ Enfermeira Residente em Cuidados Intensivos do Adulto da UEL.

${ }^{4}$ Enfermeiro. Especialista em Centro Cirúrgico e Central de Material e Esterilização. Mestrando pelo Programa de Pós-Graduação em Enfermagem da UEL.

${ }^{5}$ Enfermeira. Doutora em Enfermagem. Professora do Departamento e do Programa de Pós-Graduação em Enfermagem da UEL. Enfermeira. Doutora em Ciências da Saúde. Professora do Curso de Graduação e do Programa de Pós-Graduação em Enfermagem da UEL.
}

Autor correspondente:

Maikon Rosa dos Santos

Universidade Estadual de Londrina

R. Nossa Senhora de Lourdes, 391 - 86027-420 -Londrina-PR-Brasil

E-mail: maikon_xxx@yahoo.com.br
Recebido: 31/01/2012

Aprovado: 10/07/2012

Cogitare Enferm. 2012 Jul/Set; 17(3):524-30 


\section{INTRODUÇÃO}

A Lei 8080 de 1990 disciplina a descentralização política-administrativa do Sistema Único de Saúde que tem, entre outros objetivos, a Saúde do Trabalhador (ST) é um conjunto de atividades desenvolvidas pela vigilância epidemiológica e sanitária ${ }^{(1)}$. Ela se insere na saúde pública e visa à análise e intervenção nas relações entre o trabalho e a saúde, por meio de programas que avaliem os riscos e as condições do trabalho, proporcionando a saúde integral ao trabalhador com qualidade de vida e de trabalho, até procedimentos diagnósticos, tratamento e reabilitação nos casos de agravos e/ou doenças ${ }^{(2)}$. O mundo do trabalho é complexo e envolve determinantes que influenciam a ST, como economia, sociedade e tecnologia. Além desses, há características do ambiente de trabalho que influenciam diretamente no processo laborativo como os riscos ocupacionais que podem ser químicos, mecânicos, físicos, biológicos ou ergonômicos ${ }^{(3)}$.

Para estabelecer as diretrizes para a ST, em empresas públicas e privadas, o Ministério do Trabalho e Emprego (MTE) aprovou, em 1978, as Normas Regulamentadoras (NR) que constavam de 28 normas. A partir de então foram propostas e aceitas outras NR. Entretanto, por 27 anos, não houve por parte do MTE um programa específico de normatização para a prevenção e promoção da ST da área da saúde. Em 2005, foi aprovada uma norma específica para a área da saúde, a NR-32, que estabelece as diretrizes para a implementação de programas de prevenção de riscos ocupacionais e de proteção a ST no ambiente de trabalho ${ }^{(4-5)}$.

Esta Norma é de fundamental importância para a ST, pois a perda da capacidade laboral, e os afastamentos temporários ou permanentes do trabalho são consequências das condições insatisfatórias dos locais de trabalho ${ }^{(6)}$. A aderência à Norma proporciona benefícios para os prestadores de serviço, por meio do aumento da produtividade em função da diminuição de doenças e agravos, contribuindo para diminuir as taxas de absenteísmo decorrentes de acidentes de trabalho. Para a implantação da NR-32 são necessários recursos físicos e materiais, além de treinamentos específicos e motivação dos funcionários e administradores para a aquisição de novos comportamentos e cultura ${ }^{(7)}$. Neste contexto, é imprescindível a aplicação dos preceitos da Norma como prática diária para prevenir doenças, agravos e promover a saúde. Por ser uma nova norma de importância para a ST e cientes que os profissionais de saúde em especial os da enfermagem estão expostos a riscos advindos do meio ambiente de trabalho, este estudo teve como objetivo verificar a implantação da NR-32 em um Hospital do Norte do Paraná.

\section{MÉTODO}

Estudo descritivo, exploratório sob a forma de estudo de caso com abordagem qualitativa, desenvolvido em um Hospital Universitário. Os dados foram coletados em junho de 2010. O projeto de pesquisa foi aprovado pelo Comitê de Ética e Pesquisa da Universidade Estadual de Londrina (UEL) sob o parecer n. 076/10.

A pesquisa foi desenvolvida com base em um roteiro para elaboração de um estudo de caso clínico com as seguintes etapas: a primeira etapa foi a elaboração da questão norteadora e do check list. A questão norteadora foi elaborada para apreender como são desenvolvidas as ações para a implantação da NR-32 no hospital em estudo; o check list foi elaborado baseado nas exigências da NR-32. A segunda etapa foi a coleta de informações, nesta etapa com base check list pré-estabelecido e realizada uma entrevista gravada com a enfermeira da Comissão de Controle de Infecção Hospitalar (CCIH), posteriormente as informações foram transcritas na íntegra. A entrevistada concordou em participar do estudo, assinando o Termo de Consentimento Livre e Esclarecido. A terceira etapa consistiu em analisar as informações e categorização dos dados; o check list foi preenchido pelos pesquisadores, de acordo com as respostas fornecidas pela participante da pesquisa; a quarta etapa foi realizada com a finalidade de comparar e argumentar os achados de acordo com a literatura científica para responder a questão norteadora do estudo. A quinta etapa deu-se através da caracterização das alternativas baseadas na literatura científica para os problemas encontrados com a recomendação de ações: nesta fase foram descritas as alternativas escolhidas para modificar ou minimizar os problemas encontrados, além de justificar o motivo da escolha das propostas; e como etapa final, foi constituída da apresentação e discussão dos resultados do estudo ${ }^{(8)}$.

\section{RESULTADOS}

\section{Categoria 1 - Dificuldades de cuidados à Saúde do Tra- balhador relacionadas à negligencia dos profissionais}

Uso incorreto dos Equipamentos de Proteção Individual (EPI); agulhas dentro das enfermarias nos balcões de apoio; transporte inadequado de materiais perfurocortantes; caixas de perfurocortante com capacidade além do preconizado, com agulhas transbordando; falha na identificação dos produtos químicos; reutilização das embalagens de produtos químicos; 
utilização inadequada do impresso de Comunicação de Acidente de Trabalho (CAT) e utilização de calçados abertos por trabalhadores.

\section{Categoria 2 - Dificuldades de cuidados à Saúde do Trabalhador relacionadas à instituição}

Falta de sacos plásticos e de recipientes adequados para descarte de resíduos hospitalares; falta de manutenção de máquinas e equipamentos; falta de ventilação em ambientes; infiltração hidráulica em ambientes e localização inadequada do refeitório.

\section{DISCUSSÃO}

\section{Dificuldades de cuidados à Saúde do Trabalhador relacionadas à negligência dos profissionais}

O processo de trabalho da equipe de enfermagem, na maioria dos setores, é de assistência integral, ou seja, o mesmo profissional cuida de um grupo de pacientes, administrando medicamentos e realizando cuidados. Desta maneira, materiais e equipamentos são deixados nas enfermarias para evitar o desgaste físico devido às idas e vindas constantes do trabalhador ao posto de enfermagem. Assim sendo, agulhas nos balcões das enfermarias são encontradas.

Aliado a isso, é comum encontrar caixas de descarte repletas além dos $3 / 4$ preconizados pela Vigilância Sanitária. Vale ressaltar que estas caixas de descarte devem ficar nos postos de enfermagem. Desta maneira, os materiais perfurocortantes são transportados inadequadamente das enfermarias até o local correto de descarte, sendo transportados em bandejas, junto com os outros materiais de uso diário e muitos auxiliares/ técnicos de enfermagem utilizam os EPI incorretamente. $\mathrm{O}$ uso inadequado de embalagem de perfurocortantes propicia acidentes com materiais biológicos que podem transmitir doenças, como o vírus da imunodeficiência humana (HIV), Hepatite B e C entre os trabalhadores que atuam nos serviços de saúde ${ }^{(9)}$.

Em estudo realizado com a equipe multiprofissional de atendimento pré-hospitalar em Belo Horizonte, Minas Gerais detectou-se importante grau de desconhecimento das medidas de Proteção Padrão (PP) e relato de atitudes inadequadas, principalmente entre os condutores dos veículos e auxiliares/técnicos de enfermagem. No referido estudo nenhum profissional atingiu o percentual desejável $(\geq 75 \%)$ para uso de EPI (máscara facial e óculos de proteção) além da uti- lização adequada dos mesmos durante o atendimento. Um fator relevante é que os enfermeiros atingiram o menor percentual de atitudes corretas para indicação de uso destes equipamentos. Para facilitar a adoção das Precauções Padrão (PP) foi citado o treinamento sobre riscos ocupacionais, uso adequado de EPI e noções básicas sobre infecções, além de reuniões periódicas para facilitar entrosamento entre os membros da equipe ${ }^{(10)}$.

Enfatiza-se a importância dos fatores organizacionais que favoreçam a adoção das PP, quais sejam, o suprimento e disponibilidade adequados de EPI, a supervisão e o feedback do uso das PP e políticas que apóiem a segurança, como por exemplo, o registro e acompanhamento do acidentado, incluindo a profilaxia pós-exposição e disponibilidade de vacinação contra o vírus da hepatite $\mathrm{B}^{(11)}$.

Os resíduos do serviço de saúde são classificados de acordo com o risco em cinco grupos (A, B, C, D e E). O grupo A engloba os resíduos com possível presença de agentes biológicos que, por suas características, podem apresentar risco de infecção. Este grupo apresenta cinco subdivisões sendo que os materiais perfurocortantes ou escarificantes, entre outros, constam do subgrupo A5. O grupo B, engloba os resíduos químicos ${ }^{(12)}$. Falhas de gerenciamento de resíduos hospitalares químicos na falta de identificação clara e reutilização das embalagens para outros fins, contrariando o correto gerenciamento desses resíduos de acordo com o padronizado pela Agência Nacional Vigilância Sanitária foram observados. A NR-32 determina que todo recipiente com produto químico manipulado ou fracionado deve ser corretamente identificado, de forma legível, com etiqueta contendo: o nome do produto, composição química, sua concentração, data de validade, e nome do responsável pela manipulação ou fracionamento. A mesma norma veta a reutilização das embalagens de produtos químicos ${ }^{(5)}$.

Os riscos químicos envolvem também a manipulação de antibióticos, antineoplásicos, detergentes, gases medicinais, gases e vapores anestésicos. A inalação e contato com esses produtos podem ocasionar reações alérgicas, dificuldades respiratórias, náuseas, cefaleia e, até mesmo, abortos espontâneos ${ }^{(13)}$. De acordo com a NR-32 o transporte dos resíduos deve ser realizado em sentido único, com roteiro definido, em horários não coincidentes com a distribuição de roupas, alimentos e medicamentos, períodos de visita ou de maior fluxo de pessoas.

Em estudo com trabalhadores sobre o manejo de resíduos sólidos dos serviços de saúde, percebeu-se a necessidade da constante orientação dos trabalha- 
dores e a seriedade das ações do manejo correto dos resíduos $^{(14)}$, utilizando-se as falhas encontradas para o treinamento que gerem mudanças de comportamento, minimizem os riscos qualitativos e quantitativos inerentes aos procedimentos realizados. Desta maneira, os agravos à saúde humana e ambiental podem ser minimizados, facilitando-se a segregação dos resíduos dos serviços de saúde e melhor conhecimento da realidade para estabelecer metas e prioridadesna busca de melhorias tanto para o trabalhador como para a instituição de saúde ${ }^{(6)}$.

Quanto à exposição a radiações ionizantes, a NR-32 determina que o trabalhador que realiza atividades em áreas na qual existam fontes de radiação ionizantes deve usar os EPI adequados para diminuir os risco e ter a monitorização individual de dose de radiação ionizante nos casos em que a exposição seja ocupacional $^{(5)}$. Durante os exames de Raio-X e tomografia computadorizada, além do uso do dosímetro devem ser utilizados aventais de chumbo, entretanto, os trabalhadores deste setor, muitas vezes não valorizam e não utilizam esse EPI existente e necessários à execução de suas atividades laborais ${ }^{(15)}$, além do que não há sinalização adequada das áreas de instalação radioativas, preconizadas na NR-32.

Outra importante ação para as intuições é a notificação correta dos AT, visto que permite aos gestores das mesmas conhecer os riscos e seus agravantes e assim proporcionar medidas que venha intervir sobre os efeitos econômicos e sociais, pois esses acidentes geram um grande impacto econômico devido à perda da mão de obra qualificada pelas lesões ocupacionais, bem como propicia a diminuição da subnotificação desses agravos ${ }^{(10)}$.

\section{Dificuldades de cuidados à Saúde do Trabalhador relacionadas à instituição}

Referentes à instituição, a diversidade e quantidade de EPI, a falta de insumos (sacos de lixos e recipientes adequados para descarte), ventilação inadequada em ambientes e a ausência de programas de manutenção preventiva dos equipamentos é fato. A NR-32 preconiza que o sistema de climatização deve sofrer manutenção preventiva e corretiva para preservação da integridade e eficiência de todos os seus componentes.

Os sistemas de climatização passaram a ser um fator de satisfação e aumento da produtividade dos trabalhadores, pois o conforto térmico é um estado fisiológico o que minimiza a necessidade do sistema de termoregulação ${ }^{(16)}$. Não há manutenção preventiva dos sistemas de climatização e existe o número insuficiente de recursos humanos devido a não reposição de funcionários que estão em processo de aposentadoria, além disso, os recursos financeiros são reduzidos para as contratações. Os programas de manutenção preventiva contribuem para alcançar aumento da segurança para pacientes e trabalhadores. A redução do tempo de paralisação do equipamento e o aumento de sua vida útil, e ainda, a redução com os custos de manutenção corretiva, mas a inclusão indiscriminada de todos os equipamentos torna-se inviável financeiramente para a instituição de saúde ${ }^{(17)}$.

No campo das condições de conforto por ocasião das refeições, a NR- 32 estabelece que os estabelecimentos com 300 ou mais trabalhadores devam ser dotados de locais para refeição, com localização fora da área do posto de trabalho e possuir equipamento seguro e apropriado para o aquecimento das refeições. A instituição de estudo possui um local para refeição, no entanto, o mesmo está localizado dentro da instituição, atendendo parcialmente as prerrogativas da NR-32. Alguns equipamentos para o aquecimento das refeições são inadequados, o que propicia riscos aos trabalhadores e alteração da qualidade das refeições.

No que tange à lavanderia hospitalar o seu maior objetivo é proporcionar roupas em condições adequadas de higiene e limpeza com o intuito de oferecer segurança e conforto ao paciente ${ }^{(18)}$. O hospital em estudo realiza de maneira efetiva o que é preconizado na NR-32. Os trabalhadores que realizam a limpeza dos serviços de saúde devem ser capacitados inicialmente e de forma continuada ${ }^{(3)}$. Dessa maneira, o hospital cumpre parcialmente as recomendações da norma, por meio de atividades de educação em saúde juntamente com a CCIH e outros setores do hospital. A educação continuada é fundamental para os trabalhadores da higiene e limpeza, pois muito desses trabalhadores realizam esse serviço hospitalar pela primeira vez. Além de aprenderem a fazer uma limpeza em hospital, também aprendem como utilizar os EPI ${ }^{(19)}$.

A instituição possui um Plano de Gerenciamento de Resíduos de Serviços de Saúde conforme estabelecido no capítulo III da Resolução RDC 306/04 que preconiza a responsabilidade da segregação do lixo é do gerador dos mesmos ${ }^{(12)}$. Esse Plano é realizado juntamente com a divisão de Serviços Gerais do hospital. Entretanto, a segregação não é realizada adequadamente devido à escassez de recursos materiais, onde por vezes, faltam sacos de lixo de cores variadas 
para a correta segregação dos resíduos produzidos na instituição conforme preconizado pela Resolução RDC 306/04(12). Nesse sentido, a possibilidade de mistura do resíduo comum com o resíduo infectante é ampla, o que aumenta a proliferação de microorganismos patogênicos, gera gastos para a instituição e aumenta o risco de acidentes ocupacionais entre os trabalhadores.

\section{Adequações realizadas pela instituição e a Preven- ção dos Riscos Ocupacionais}

Foi instituída uma comissão para estudar e propor formas de implantação das determinações contidas na NR-32. Para tanto, esta comissão, por meio de negociações com a diretoria do hospital, decidiu estabelecer prioridades para a implantação. Assim, para atender as exigências da NR-32, a instituição está adequando os uniformes privativos para uso exclusivo no trabalho e que estão sendo cedidos pelo próprio hospital.

Em relação às medidas de proteção, o empregador deve vetar o uso de adornos e de calçados abertos e os trabalhadores não devem deixar o local de trabalho com EPI e/ou com as vestimentas utilizadas em suas atividades laborais ${ }^{(3)}$. Nesta instituição, muitas vezes não é vetado o uso de adornos por parte daqueles que prestam assistência à saúde. Recomenda-se ainda criar uma cartilha com itens obrigatórios para uma adequada postura profissional nos locais de serviços de saúde, contemplando a diminuição do uso de adornos entre outros. A NR-32 refere que o Programa de Prevenção de Riscos Ambientais (PPRA) deve conter a identificação dos riscos mais prováveis e a avaliação do local de trabalho e do trabalhador. A identificação dos riscos engloba: fontes de exposição e reservatório; vias de administração; transmissibilidade, patogenicidade e virulência do agente; persistência do agente biológico no ambiente; estudos epidemiológicos ou dados estatísticos e outras informações científicas. Já a avaliação engloba a finalidade e descrição do local de trabalho, sua organização e procedimento, a possibilidade de exposição, a descrição das atividades e funções de cada local e as medidas preventivas aplicáveis, e seu acompanhamento $^{(11)}$.

O PPRA deve ser reavaliado uma vez ao ano ou sempre que ocorram mudanças nas condições de trabalho que alterem a exposição dos trabalhadores aos riscos ocupacionais ${ }^{(3)}$. Destaca-se que a enfermeira entrevistada não abordou este fato em sua entrevista, provavelmente porque este Programa é de responsabilidade do Serviço de Segurança e Medicina do Tra- balho. O Programa tem como objetivos a prevenção e controle do trabalhador em relação aos riscos físicos, químicos e biológicos presentes nos locais de trabalho; deve ser desenvolvido em cada estabelecimento sob a responsabilidade do empregador e com a participação dos trabalhadores ${ }^{(11)}$.

O Programa de Controle Médico de Saúde Ocupacional é estabelecido pela NR-7, que deve ser de caráter preventivo, realizando prevenção e diagnóstico médico dos agravos a ST. Além do previsto na NR-7, a NR-32 determina que todo acidente de trabalho envolvendo riscos biológicos, com ou sem afastamento do trabalhador, necessita da emissão de CAT. O acidente é, muitas vezes, subnotificado pelo empregador, profissional ou pelo próprio acidentado ${ }^{(20)}$. Deste modo, percebe-se que na instituição em estudo não é diferente. Ainda os trabalhadores muitas vezes acabam desvalorizando o preenchimento de CAT, perdem o amparo legal e se expõem aos riscos de adquirirem uma doença em decorrência do acidente. Neste sentido, os protocolos de prevenção e controle da ST na instituição devem ser revistos e efetivamente aplicados para que os trabalhadores tenham a exposição aos riscos melhor controlada.

\section{CONSIDERAÇÕES FINAIS}

Verifica-se que apesar das dificuldades encontradas na instituição, a mesma já adere certos quesitos em relação à NR-32 em processo de implementação ou na sua totalidade. Acredita-se que durante o processo de mudança da norma vigente para a NR-32, mudanças benéficas serão alcançadas, uma vez que medidas protetoras e procedimentos deverão ser cumpridos com vistas à prevenção de $\mathrm{AT}$ e, por sua vez promover segurança no trabalho e uma melhor assistência ao paciente.

Ressalta-se que o impacto financeiro causado pela necessidade de recursos físicos e materiais gera altos custos, que muitas vezes causam limitações para os serviços de saúde na implantação da NR-32. Entretanto, enfatiza-se que acrescentar qualquer quantia financeira aplicada em favor da NR-32 terá um reflexo positivo no futuro, garantindo assim ganho social na prevenção de morbidades relacionadas ao trabalho.

Identificou-se que a instituição em estudo, muitas vezes não veta o uso de adornos, e que os trabalhadores não utilizam corretamente o EPI, o que propicia a ocorrência de AT. Ainda percebeu-se que muitas vezes os trabalhadores desvalorizam o preenchimento de CAT, o que propicia que os mesmos se exponham aos riscos de adquirirem uma doença, além de perderem o amparo legal. 
Destaca-se que a adesão correta aos EPI é essencial para uma assistência de qualidade, sendo fundamental a conscientização da equipe sobre os procedimentos a serem adotados e a importância da utilização desses equipamentos, bem como, a educação continuada. Apesar de a instituição ter ações educativas e medidas preventivas, ainda observa-se que acontecem muitos AT no referido hospital e que na maioria das vezes estão relacionados aos próprios trabalhadores que não seguem as normativas da instituição.

Coloca-se aqui a limitação deste estudo de caso fazendo-se necessário um estudo mais aprofundado que busque diversos pontos de vistas dos trabalhadores, visto que os mesmos vivenciam diariamente as dificuldades e limitações em relação à utilização da NR-32 no hospital em estudo.

\section{REFERÊNCIAS}

1. Brasil. Lei no ${ }^{\circ}$ 8080, de 19 de setembro de 1990. Dispõe sobre as condições para promoção e recuperação da saúde, a organização e o funcionamento dos serviços correspondentes e dá outras providências. Presidência da República Casa Civil Subchefia para Assuntos Jurídicos Brasília, DF, [internet] 19 set. 1990. [acesso em 12 maio 2012]. Disponível: http://www.planalto.gov. br/ccivil_03/leis/18080.htm.

2. Ministério da Saúde (BR). Organização Panamericana de Saúde (OPAS). Organização Mundial de Saúde (OMS). Doenças relacionadas ao trabalho: manual de procedimentos para serviços de saúde. Brasília; 2001. [acesso em 11 ago 2011]. Disponível: http://dtr2001. saude.gov.br/editora/produtos/livros/pdf/02_0388 M1.pdf

3. Robazzi MLCC, MarzialeMH. Anorma regulamentadora 32 e suas implicações sobre os trabalhadores de enfermagem. Rev. Latino-Am. Enfermagem. [Internet]. 2004;12(5). [acesso em 11 ago 2011] Disponível: http:// www.bvsde.paho.org/bvsacd/cd49/v12n5a19.pdf

4. Sarquis LMM, Cruz EBS, Hausmann M, Felli VEA, Peduzzi M. Uma reflexão sobre a saúde do trabalhador de enfermagem e os avanços da legislação trabalhista. Cogitare enferm. [Internet]. 2005;9(1). Disponível: http://ojs.c3sl.ufpr.br/ojs2/index.php/cogitare/article/ view/1701/1409

5. Brasil. Portaria n. 219, de 16 nov. 2005. Aprova a Norma Regulamentadora NR 32 Segurança e Saúde no Trabalho em Estabelecimentos de Saúde. Diário Oficial da União, Brasília, n.219, 16 nov. 2005. Seção 1.
6. Santos L B. Resíduos dos serviços de saúde: diagnóstico e riscos no Centro de Quimioterapia do Hospital de Referência de Araguaína (TO). [Dissertação]. Goiânia (GO): Universidade Católica de Goiás; 2009.

7. Fagundes G. NR-32 uma realidade na área hospitalar. [Internet] [acesso em 27 mar 2010]. Disponível: http://www. saudeetrabalho.com.br/download/NR32-gilmara.pdf

8. Galdeano LE, Rossi LA, Zago MMF. Roteiro instrucional para a elaboração de um estudo de caso clínico.[Internet]. Rev. Latino-Am. Enfermagem. 2003;11(3). [acesso em 27 mar 2011]. Disponível: http:// www.scielo.br/pdf/rlae/v11n3/16548.pdf.

9. Marziale MHP, Nishimura KYN, Ferreira MM. Risco de contaminação ocasionados por acidentes de trabalho com material perfurocortantes entre trabalhadores de enfermagem. Rev. Latino-Am. Enfermagem. 2004;12:36-42.

10. Lopes ACS, Oliveira AC, Silva JT, Paiva MHRS. Adesão às preocupações padrão pela equipe de enfermagem pré-hospitalar móvel de Belo Horizonte, Minas Gerais Brasil.2008 Cad. saude publica. [Internet]. 24(6):1387-96 [acesso em 27 mar 2011] . Disponível: ttp://www.scielo.br/pdf/csp/v24n6/19.pdf

11. Brevidelli MM, Cianciarulo TI. Níveis de adesão às precauções-padrão entre os profissionais médicos e de enfermagem de um hospital universitário. Online braz. j. nurs. [Internet] 2006; 5(1) [acesso em 27 mar 2011] Disponível: http://bases.bireme.br/cgi-bin/wxislind.exe/ iah/online/?IsisScript $=$ iah/iah.xis $\& s r c=$ google $\&$ base $=$ LILACS\&lang $=$ p\&nextAction $=$ lnk\&exprSearch $=4902$ 23\&indexSearch $=$ ID

12. Brasil. ANVISA. A Diretoria Colegiada da Agência Nacional de Vigilância Sanitária. Aprova o regulamento técnico para o gerenciamento de resíduos de serviço de saúde. Resolução-RDC n. 306, [Internet] 7 dez. 2004. [acesso em 27 mar 2011]. Disponível: http://www.iec. pa.gov.br/cibio/arquivos/RDC306-2004NVISA.pdf

13. Xelegati R, Robazzi MLCC. Riscos químicos a que estão submetidos os trabalhadores de enfermagem: uma revisão de literatura. Rev. Latino-Am. Enfermagem. 2003;11(3):350-6.

14. Dói KM, Moura GMSS. Resíduos sólidos do serviço de saúde: uma fotografia do comprometimento da equipe de enfermagem. [Internet]. Rev. gauch. enferm. 2011;32(2) [acesso em 27 mar 2011]. Disponível: http:// seer.ufrgs.br/RevistaGauchadeEnfermagem/article/ viewFile/14802/12781 
15. Fernandes GS, Carvalho ACP, Azevedo ACP. Avaliação dos riscos ocupacionais de trabalhadores de serviços de radiologia. 2005. Radiol. Bras. 2005;38(4):279-81.

16. Fialho FAP, Silva LB. Contribuição para validação de um modelo teórico de conforto termoambiental. PS Periódico Saúde Paraíba. 2000;1(5):17-32.

17. Morais VC. Metodologia de priorização de equipamentos médico-hospitalares em programas de manutenção preventiva. [dissertação]. Campinas (SP): UNICAMP; 2004

18. Cargnin MT. Análise do processo de lavagem e desinfecção de roupas hospitalares: o caso da lavanderia HUSM. [dissertação]. Santa Maria (RS): Universidade Federal de Santa Maria; 2008.

19. Monteiro MI, Chillida MSP, Bargas EB. Educação continuada em um serviço terceirizado de limpeza de um hospital universitário. Rev. Latino-Am. Enfermagem. [Internet] 2004;12(3) [acesso em 27 mar 2011]. Disponível: http://www.scielo.br/pdf/rlae/v12n3/ v12n3a13.pdf

20. Silveira CA, Robazzi MLCC. A subnotificação acidentária entre os trabalhadores rurais em um hospital público. In: Anais do $17^{\circ}$ Simpósio Internacional de Iniciação Científica da USP; [Internet]. 2003 [acesso em 30 jul 2012]. Disponível: https://uspdigital.usp.br/ siicusp/cdOnlineTrabalhoObter?numeroInscricaoTrab alho $=752 \&$ numeroEdicao $=11 \&$ print $=S$ 